\title{
Fixed-Order $\mathscr{H}_{\infty}$ Controller Design via Convex Optimization Using An Alternative to Youla Prameterization
}

\author{
Hamid Khatibi and Alireza Karimi
}

\begin{abstract}
All $\mathscr{H}_{\infty}$ controllers of a SISO LTI system are parameterized thanks to the relation between Bounded Real Lemma and Positive Real Lemma and a new concept of strict positive realness of two transfer functions with the same Lyapunov matrix in the matrix inequality of the Kalman-Yakubovic-Popov lemma. This new parameterization shares the same features with Youla parameterization, namely on the convexity of $\mathscr{H}_{\infty}$ norm constraints for the closed-loop transfer functions. However, in contrary to Youla parameterization, it can deal with any controller order and any controller structure such as e.g. PID. The main feature of the proposed method is that it can be extended easily for the systems with polytopic uncertainty. This way, a convex inner approximation of all $\mathscr{H}_{\infty}$ controllers for polytopic systems is given, which can be enlarged by increasing the controller order. In order to design a low-order robust $\mathscr{H}_{\infty}$ controller with less conservatism, rank of the $k$-th Sylvester resultant matrix of the controller is made to be deficient via a convex approximation of the rank minimization problem. The effectiveness of the proposed method is shown via simulation results.
\end{abstract}

\section{INTRODUCTION}

Youla parameterization [1] is probably the most well-known controller parameterization, which parameterizes all stabilizing controllers of a system, over an infinite dimensional space. The main advantage of this parameterization is that all closed-loop sensitivity functions are affine w.r.t. the so-called $Q$ parameter and hence, it can be employed for $\mathscr{H}_{\infty}$ controller design in a convex

The authors are with the Laboratoire d'Automatique of Ecole Polytechnique Fédérale de Lausanne (EPFL), 1015 Lausanne, Switzerland.

Corresponding author: alireza.karimi@epfl.ch 
optimization problem. It has two major disadvantages. First, it depends on system parameters and therefore, it cannot be used for systems with parametric uncertainty such as polytopic systems. Second, it cannot deal with fixed-order controllers or enforce a prescribed controller structure such as e.g. PID.

Fixed-order controller design has been always a challenging problem for control engineers and has attracted many recent researchers. The research is motivated by the real-time implementation of systems with high sampling rate, where the fast computation of the command is crucial and also by many other practical applications, such as embedded control systems for the space and aeronautics industries, where the simplicity of both the code and the hardware are of great importance. Several analytical solutions are available in the literature (see e.g. [2]). Nevertheless, the main difficulty in these results is that they are not computationally efficient, which means that there do not exist fast and reliable methods to compute optimal fixed-order controllers. The main problem stems from the fundamental algebraic property that the stability domain in the space of polynomial's parameters is non-convex for polynomials with order higher than two [3]. This problem can be formulated as Bilinear Matrix Inequality (BMI) [4] that has been shown to be generally NP-hard [5]. In [6] the authors parameterize all stabilizing controllers via two LMI conditions where the controller order can be fixed by imposing a rank condition at the expense of convexity. Some designers prefer to solve a suboptimal convex problem rather than the nonconvex problem [7], [8], [9], [10]. Nonetheless, several researches have been accomplished to solve the non-convex problem in special cases. In [11], a non-linear algorithm is adopted which converges, under mild conditions, to a point that satisfies only the first order necessary conditions of optimality. Another way to solve this non-convex problem is to gather all the non-convexity in a rank constraint [12], [13].

Rank minimization is a challenging issue in the control engineering. Although it has been shown that many control problems can be reduced to a rank minimization problem (see [14] and the references therein), the existing convexified rank minimization methods are more heuristic than rigorous and hence, not so efficient. In this paper, the problem is formulated such that a Sylvester matrix should be just rank-deficient and there is no need to minimize its rank. That is, using an appropriate weighting, only one of the eigenvalue is minimized to make the matrix rank deficient. To form a convex problem, the approximation proposed by [15] is used and its efficiency is improved by using a weighted trace function instead of the regular one. 
The problem of fixed-order controller design becomes more complicated when a fixed-order controller should stabilize a system with polytopic uncertainty.

Polytopic representation is one of the most general ways of capturing physical parameter uncertainty, multi-model systems and the well-known interval systems, and has attracted many robust controller designers recently. Robust stability of polytopic systems is analyzed either in the polynomial form using the generalized Kharitonov theorem [16] or in the state space using the concept of quadratic stability, where a Lyapunov function should be found for all vertices of the polytopic system. However, this approach suffers considerably from conservatism [17]. A less conservative approach is the use of a parameter dependent Lyapunov function, which leads to a sufficient condition for the robust stability analysis, represented by LMIs [18], [19], [20], [21].

Although robust stability analysis for polytopic systems has been adequately studied in the literature, the results for robust control synthesis for these systems are rather limited. A classical approach is $\mu$ synthesis which leads to a non-convex optimization problem [22]. The convex optimization solutions are generally based on the quadratic stability of the whole polytope using a Lyapunov function which turns out to be too conservative. In most of the early approaches, a state feedback controller is designed based on the parameter dependent Lyapunov approach and the use of convex optimization algorithms via LMIs [23], [24]. To the best of our knowledge, the only approaches that do not consider the quadratic stability for the output feedback design of polytopic systems and use a convex optimization algorithm are mainly given by [25], [8] and [26], where the principal idea comes from the strictly positive realness (SPRness) of transfer functions. A synthesis method is proposed by [25], which can consider a specific "rank one" type of uncertainty that covers the polytopic uncertainty with some conservatism. Besides, since a Q-parameterization method is involved in the synthesis approach, fixed-order controller design cannot be handled. In addition, robust performances do not become convex w.r.t. their parameterization. In [8] a convex parameterization of fixed-order stabilizing controllers for polytopic systems based on the polynomial positivity is given. The approach gives only a stabilizing controller (a feasible point), which relies on a so-called central polynomial. The same method is used for convex parameterization of fixed-order $\mathscr{H}_{\infty}$ controllers in [27]. Yet the solution depends on the choice of the central polynomial. The effect of this choice is studied in [10], where a robust regional pole-placement is performed by a proper choice of this polynomial. 
On the other hand, the utilized LMIs in [10] are originated from the Kalman-Yakubovic-Popov (KYP) lemma instead of polynomial positivity, which can be solved very efficiently using the existing LMI solvers [28]. Albeit, all these approaches suffer from the conservatism imposed by fixing a polynomial and hence if the proposed optimization problem becomes infeasible, it is not possible to conclude that there does not exist a stabilizing controller of the desired order for the uncertain system. From another point of view, in these approaches, a convex inner approximation of the non-convex set of all fixed-order stabilizing controllers is developed, which suffers from conservatism.

In a similar approach, it is shown that the dependence on the central polynomial can be relaxed by increasing the controller order, where a new parameterization of all stabilizing controllers in an infinite dimensional space is given [26]. The main advantage of the results in [26] with respect to the methods of [8] and [10] is that the given parameterization covers all stabilizing controllers in an infinite dimensional space, whose inner approximation for fixed-order controllers coincides with the results of [8] and [10]. The advantage of this parameterization w.r.t. Youla parameterization is that it parameterizes all stabilizing controllers of a polytopic system and in addition, it can deal with any controller structure and a prescribed order. Furthermore, in contrary to Youla parameterization, it does not lead to affine closed-loop transfer functions w.r.t. the controller parameters. In order to convexify the norm constraints on the closed-loop transfer functions, appropriate norm constraints are imposed on their numerator and denominator separately. This separation method for convexification is also utilized in [7].

An alternative to Youla parameterization of all $\mathscr{H}_{\infty}$ controllers is given in this paper. The main advantage of this new parameterization is that it can enforce any controller structure and order. Withal, similar to Youla parameterization and in contrary to the parameterization of [26], all closed-loop transfer functions become affine w.r.t. the variables, which enables a convex parameterization of all $\mathscr{H}_{\infty}$ controllers. Besides, it can be easily employed for polytopic systems to give a convex inner approximation of all $\mathscr{H}_{\infty}$ controllers. The problem considered in this paper is very similar to that of [27], where a convex parameterization of fixed-order $\mathscr{H}_{\infty}$ controllers is given using the properties of positive polynomials and Finsler's lemma. However, in this paper a different approach based on a proposed concept of strict positive realness of two transfer functions with the same Lyapunov matrix in the matrix inequality of the KYP lemma is employed. It should be emphasized that there is no conservatism for systems without uncertainty and for polytopic 
systems it can be reduced by increasing the order of the controller. In addition, for continuoustime systems it is shown that it is possible to minimize the desired $\mathscr{H}_{\infty}$ norm, whereas in [27], the iterative bisection algorithm should be used to find the minimum value of the desired $\mathscr{H}_{\infty}$ norm. Along with, a new constraint is employed in order to obtain a low-order controller. This constraint is a rank deficiency constraint on the Sylvester resultant matrix of the numerator and denominator polynomials of the controller. Since this constraint is not convex, some convex approximations based on trace minimization are introduced and compared.

The approach of this paper is comparable to that of [26]. However, the approach of [26] has two major disadvantages that are resolved in this paper. First, it may result in a highorder controller, which is not acceptable for many real systems. This problem is dealt with in this paper, where a rank-deficiency constraint on the $k$-th Sylvester matrix of the controller causes the algorithm to come up with a controller that has a predetermined number of pole-zero cancellation. Second, the proposed convex approximation for performance specifications is not efficient such that minimizing the suggested cost-function or increasing the controller order, do not necessarily result in better performance specifications. To obtain a better performance, a new convex approximation is proposed in this paper, which works quite efficiently. Further, the new constraint contains the stability criterion inside and hence, there is no extra constraint for the stability as in [26].

The rest of the paper is organized as follows. The notation and some basic results are recalled in Section II. Section III introduces the new convex constraints that satisfy $\mathscr{H}_{\infty}$ performance specifications. Rank-deficiency constraint is introduced in Section IV. Concluding remarks are given in Section V. Finally, it is shown in the Appendix that in case of continuous-time systems, it is possible to minimize the desired $\mathscr{H}_{\infty}$ norm in a convex optimization problem.

\section{PRELIMINARIES}

A finite order rational transfer function of a SISO LTI plant in discrete- or in continuous-time is represented by its coprime factors $N$ and $M$ :

$$
G=N M^{-1}, \quad N, M \in \mathscr{R} \mathscr{H}_{\infty}
$$

where $\mathscr{R} \mathscr{H}_{\infty}$ is the set of proper stable rational transfer functions with bounded infinity norm. The denominators of $N$ and $M$ are supposed to be identical and their numerators are referred to 
as numerator and denominator polynomials of $G$, respectively. Furthermore, a polytopic system is defined with its $q$ vertices such that the $i$-th vertex consists of the parameters of the model $G_{i}=N_{i} M_{i}^{-1}$, where $N_{i}$ and $M_{i} \in \mathscr{R} \mathscr{H}_{\infty}$ are the coprime factors of $G_{i}$. Thus, the whole polytope can be shown by :

$$
\mathscr{G}:\left\{G=N M^{-1} \mid N=\sum_{i=1}^{q} \lambda_{i} N_{i}, M=\sum_{i=1}^{q} \lambda_{i} M_{i}\right\}
$$

where $\lambda_{i} \geq 0$ and $\sum_{i=1}^{q} \lambda_{i}=1$. A controller is represented by $K=X Y^{-1}$, where $X, Y \in \mathscr{R} \mathscr{H}_{\infty}$ are not necessarily coprime.

Unless stated, all the state space realizations $(A, B, C, D)$, are supposed to be in controllable canonical form and therefore, $A$ is only related to the denominator, $C$ and $D$ are affinely affected by both the denominator and the numerator, and $B$ is fixed.

The proposed method of this paper covers both discrete-time and continuous-time systems, but for simplicity wherever there is a difference between their representations, the equations are given for discrete-time systems. The main difference stems from the Lyapunov inequality and the inequality of the Kalman-Yakubovic-Popov (KYP) lemma :

Lemma 1: (the KYP lemma [29] for discrete-time systems) A biproper transfer function $H(z)=$ $C(z I-A)^{-1} B+D$ belongs to $\mathscr{S}$, where $\mathscr{S}$ is the convex set of all Strictly Positive Real (SPR) transfer functions, if and only if there exists a matrix $P=P^{T}>0$ such that :

$$
\left[\begin{array}{cc}
A^{T} P A-P & A^{T} P B-C^{T} \\
B^{T} P A-C & B^{T} P B-D-D^{T}
\end{array}\right]<0
$$

It is evident that if the denominator of $H(z)$ is fixed, the above matrix inequality becomes an LMI, since the variables $P, C$ and $D$, appear affinely in it.

\section{Parameterization of All $\mathscr{H}_{\infty}$ CONTROLlers}

To ensure the robust performance, we want to parameterize all stabilizing controllers that satisfy some $\mathscr{H}_{\infty}$ norm bounds on some weighted transfer functions of the closed-loop system. However, for the simplicity of the equations, we demonstrate the method with $\mathscr{H}_{\infty}$ norm bound on only one sensitivity function. Thus, without loss of generality, suppose that it is desired to have :

$$
\left\|W_{1} S\right\|_{\infty}=\left\|\frac{W_{1} M Y}{M Y+N X}\right\|_{\infty}<\gamma
$$


for a given $\gamma$. It is well-known that an infinity norm constraint could be presented as LMIs via Bounded Real Lemma, if the denominator of its argument is fixed [30]. However, in (4), the controller parameters appear both in numerator and denominator of $W_{1} S$, which results in a BMI problem. In order to convexify this performance constraint, the relation between Bounded Real Lemma and Positive Real Lemma is employed. It is well-known that (4) is equivalent to the SPRness of [31] :

$$
\frac{(M Y+N X)-\gamma^{-1} W_{1} M Y}{(M Y+N X)+\gamma^{-1} W_{1} M Y}
$$

Therefore, the set of all controllers that result in a closed-loop system with $\left\|W_{1} S\right\|_{\infty}<\gamma$ for a system $G$ defined in (1), is given by :

$$
\begin{aligned}
\mathscr{K}_{\infty}:\{ & K=X Y^{-1} \mid \\
& \left.\frac{(M Y+N X)-\gamma^{-1} W_{1} M Y}{(M Y+N X)+\gamma^{-1} W_{1} M Y} \in \mathscr{S}\right\}
\end{aligned}
$$

where $X, Y \in \mathscr{R} \mathscr{H}_{\infty}$. Using the KYP lemma (Lemma 1 for discrete-time systems), the SPRness of a transfer function with fixed denominator can be represented via LMIs. However, in (6), both numerator and denominator contain optimization variables and hence, the set is not convex in this form.

In the sequel, (6) is represented via LMIs. Moreover, it is shown that the resulting LMIs give the complete set of all stabilizing $\mathscr{H}_{\infty}$ controllers.

\section{A. LMI representation}

The following definitions and lemmas are required to proceed.

Definition 1: A matrix $A$ is called the state space matrix of a monic polynomial $p$, if $A$ is the controllable canonical state space matrix of the transfer function $1 / p$.

Definition 2: Consider two equal-order monic polynomials $p_{1}$ and $p_{2}$ and their state space matrices $A_{1}$ and $A_{2}$, respectively. Then, $p_{1}$ and $p_{2}$ (also $A_{1}$ and $A_{2}$ ) are called Common Lyapunov stable, or CL-stable, if $A_{1}$ and $A_{2}$ satisfy Lyapunov inequality with the same Lyapunov matrix $P$, namely for discrete-time systems $\exists P=P^{T}>0$ such that :

$$
A_{1}^{T} P A_{1}-P<0 \text { and } A_{2}^{T} P A_{2}-P<0
$$

Lemma 2: [32] A transfer function $H$ is SPR if and only if its numerator and denominator are CL-stable. 
Definition 3: Two equal-order SPR transfer functions $H_{1}$ and $H_{2}$ with controllable canonical state space realizations $\left(A_{1}, B_{1}, C_{1}, D_{1}\right)$ and $\left(A_{2}, B_{2}, C_{2}, D_{2}\right)$ are called Common Lyapunov Strictly Positive Real, or CL-SPR, if both satisfy the inequality of the KYP lemma (Inequality (3) for discrete-time systems) with the same Lyapunov matrix $P$.

Remark : A very simple consequence of the above definition is that an SPR transfer function $H_{1}$ is CL-SPR with all positive fix transfer functions such as $H_{2}=1$.

Lemma 3: An SPR transfer function $H$ and its inverse $H^{-1}$ are CL-SPR.

Proof: Using Schur complement on (3) for both $H$ and its inverse, the proof is obtained easily. Furthermore, the proof is similar for continuous-time systems.

Since the inequality of the KYP lemma contains the Lyapunov stability constraint in its first block, Lemma 3 covers Lemma 2.

The following corollary shows how a CL-SPR constraint results in a CL-stability constraint :

Corollary 1: If two transfer functions $H_{1}$ and $H_{2}$ are CL-SPR then all of their numerator and denominator polynomials are CL-stable.

Proof: Suppose that there exists $P=P^{T}>0$ satisfying the inequality of the KYP lemma for both transfer functions $H_{1}$ and $H_{2}$. Since the first block of this LMI is the same as the Lyapunov stability criterion, the denominators of these two transfer functions are CL-stable. Furthermore, according to Lemma 3, the same matrix $P$ satisfies the LMI of the KYP lemma for $H_{1}^{-1}$ and $H_{2}^{-1}$, which means that the same $P$ satisfies Lyapunov stability criterion for the numerators of $H_{1}$ and $H_{2}$. Hence, all of the four polynomials are CL-stable with the same matrix $P$.

Remark : Any $P=P^{T}>0$ that reveals the CL-stability of two polynomials, does not necessarily satisfy the LMI of the KYP lemma for their SPR ratio.

According to Lemma 2, we need to have CL-stability between numerator and denominator polynomials of (6) to prove its SPRness. However, the SPRness constraint of (6) becomes a non-convex inequality due to the existence of variables in its denominator, which causes multiplication of variables in the first block of the inequality of the KYP lemma. Taking into account Corollary 1, it is possible to impose a CL-SPR constraint on the transfer functions of numerator and denominator of (6) instead of a CL-stability constraint on its numerator and denominator polynomials. This CL-SPR constraint brings conservatism if the denominators of the mentioned transfer functions are fixed. However, this conservatism can be removed by letting the order of the controller be increased. The following theorem proposes a new convex 
parameterization of all $\mathscr{H}_{\infty}$ controllers for a system without uncertainty :

Theorem 1: Consider the numerator and the denominator transfer functions of (6) :

$$
\begin{aligned}
& (M Y+N X)-\gamma^{-1} W_{1} M Y \\
& (M Y+N X)+\gamma^{-1} W_{1} M Y
\end{aligned}
$$

Then, the set of all stabilizing controllers that result in a closed-loop system with $\left\|W_{1} S\right\|_{\infty}<\gamma$ for a system $G$ defined in (1), is given by :

$$
\mathscr{K}_{\infty}:\left\{K=X Y^{-1} \mid(7) \text { and (8) be CL-SPR }\right\}
$$

where $X, Y \in \mathscr{R} \mathscr{H}_{\infty}$.

Proof: Sufficiency: It should be shown that any controller satisfying (9), satisfies the norm constraint (4) and stabilizes the closed-loop system too. Taking into account Corollary 1, since (7) and (8) are CL-SPR transfer functions, their numerators are CL-stable. Thus, based on Lemma 2, this controller belongs to the set represented in (6), which means that it satisfies the $\mathscr{H}_{\infty}$ constraint in (4). Furthermore, since the SPRness is a convex constraint, having two SPR transfer functions (7) and (8), results in the SPRness of their sum, which means that $M Y+N X$ is SPR. Therefore, the controller is a stabilizing controller too, according to Th. 1 of [26].

Necessity: It should be shown that if $K_{0}=X_{0} Y_{0}^{-1}$ is a stabilizing controller that satisfies (4), then it belongs to $\mathscr{K}_{\infty}$ in (9). Suppose that $X_{0}, Y_{0} \in \mathscr{R} \mathscr{H}_{\infty}$ are coprime factors of $K_{0}$. Then,

$$
\frac{\left(M Y_{0}+N X_{0}\right)-\gamma^{-1} W_{1} M Y_{0}}{\left(M Y_{0}+N X_{0}\right)+\gamma^{-1} W_{1} M Y_{0}}
$$

is SPR, but $\left(M Y_{0}+N X_{0}\right)+\gamma^{-1} W_{1} M Y_{0}$ and $\left(M Y_{0}+N X_{0}\right)-\gamma^{-1} W_{1} M Y_{0}$ are not CL-SPR. We should show that there exists always a transfer function $F$, such that (7) and (8) become CLSPR with $X=X_{0} F$ and $Y=Y_{0} F$, which means that $K_{0}=\left(X_{0} F\right)\left(Y_{0} F\right)^{-1}$ belongs to $\mathscr{K}_{\infty}$ in (9). By taking $F=\left(M Y_{0}+N X_{0}\right)+\gamma^{-1} W_{1} M Y_{0}$, (7) and (8) respectively become equal to the SPR transfer functions (10) and 1, which are CL-SPR according to the given remark after Def. 3. Hence, $K_{0}$ belongs to $\mathscr{K}_{\infty}$ in (9) with $X=X_{0} F$ and $Y=Y_{0} F$.

To design a fixed-order controller, a fixed polynomial should be chosen for the denominators of (7) and (8). It is clear that by fixing these denominators, the convex feasibility set of the CL-SPR constraint of (9) would be an inner approximation of the non-convex set of all $\mathscr{H}_{\infty}$ stabilizing controllers of the desired order. An unsuitable choice of this polynomial may cause 
a null feasibility set. This conservatism can be removed by letting the order of $X$ and $Y$ be increased. By increasing the order of $X$ and $Y$, not only some $\mathscr{H}_{\infty}$ stabilizing controllers of the new orders are included in the feasible set of the problem, but also more controllers of lower orders enter in the feasible set as can be seen by the above proof. To proceed, $X$ and $Y$ can be approximated using different types of orthonormal basis functions. For instance, consider that $X$ and $Y$ are linearly parameterized by :

$$
X=\sum_{i=0}^{m} x_{i} \beta_{i} \quad ; \quad Y=\sum_{i=0}^{m} y_{i} \beta_{i}
$$

where $\beta_{i}=1 /(z-\zeta)^{i}, i=0, \ldots, m$ are the basis functions. As a result, the CL-SPR constraint in (9) becomes linear in the parameters of $X$ and $Y$ and can be represented by LMIs thanks to the KYP lemma :

$$
\begin{aligned}
\mathscr{K}_{\infty}:\{K= & X Y^{-1} \mid P=P^{T}>0, \\
& {\left[\begin{array}{cc}
A^{T} P A-P & A^{T} P B-C_{1}^{T} \\
B^{T} P A-C_{1} & B^{T} P B-D_{1}-D_{1}^{T}
\end{array}\right]<0, } \\
& {\left.\left[\begin{array}{cc}
A^{T} P A-P & A^{T} P B-C_{2}^{T} \\
B^{T} P A-C_{2} & B^{T} P B-D_{2}-D_{2}^{T}
\end{array}\right]<0\right\} }
\end{aligned}
$$

where $\left(A, B, C_{1}, D_{1}\right)$ and $\left(A, B, C_{2}, D_{2}\right)$ are the controllable canonical state space realizations of (7) and (8), respectively. The state matrix $A$ is assumed to be identical for both realizations because the denominators of both transfer functions are the same. Besides, $B$ is always the same for controllable canonical realizations.

Using the above parameterization, any controller structure and order can be enforced, whereas in Youla parameterization it is not possible. Moreover, this parameterization can be used for systems with polytopic uncertainty.

Remark : It is possible to improve our choice of the central polynomial in few steps before running the iterative bisection algorithm. These few steps not only prepare a better choice of the central polynomial, but also provide a good choice of starting $\gamma$, which helps the algorithm to converge faster. This idea is explained in the following example :

Example 1: Consider the problem of controller design for the active suspension system, which has been a benchmark system for a special issue of the European Journal of Control on fixedorder controller synthesis [33]. The goal is to design a low-order discrete-time controller such 
that two closed-loop sensitivity transfer functions satisfy certain frequency-dependent bounds. The system is approximated by a 16th order discrete-time transfer function described in [33] with a sampling period $T_{s}=1 / 800 \mathrm{~s}$. In addition to the given frequency bounds on the closedloop transfer functions $S_{y p}=1 /(1+K G)$ and $S_{u p}=K /(1+K G)$, the controller should contain a fixed term $(z+1)$ in its numerator. Here we will design a 3rd order controller that has a zero at $z=1$. We start with the constraint on $S_{y p}$, which is more difficult to be satisfied. In order to design a suitable weighting function we have used the filter design toolbox of MATLAB. The magnitude bode diagrams of the inverse of these weighting functions and the desired constraints on $S_{y p}$ and $S_{u p}$ are depicted in Figures 1 and 2 respectively:

$$
\begin{gathered}
W_{1}=\frac{z^{8}-2.769 z^{7}+5.381 z^{6}-7.376 z^{5}+7.525 z^{4}-6.176 z^{3}+3.81 z^{2}-1.703 z+0.5665}{1.267 z^{8}-3.446 z^{7}+6.509 z^{6}-8.811 z^{5}+8.83 z^{4}-7.319 z^{3}+4.634 z^{2}-2.157 z+0.8006} \\
W_{2}=\frac{z^{7}-4.878 z^{6}+10.59 z^{5}-13.3 z^{4}+10.5 z^{3}-5.248 z^{2}+1.551 z-0.21}{10.11 z^{7}-0.2399 z^{6}+0.2165 z^{5}-0.1268 z^{4}+0.07566 z^{3}-0.02589 z^{2}+0.00302 z-5.587 e-005}
\end{gathered}
$$

Since always the numerator of $W_{1}^{-1}(M Y+N X)$ divided by central polynomial should become an SPR transfer function, it is recommended to choose the central polynomial to contain the denominator of $W_{1}$ and the desired roots of closed-loop characteristic polynomial, which is the same as the numerator of $M Y+N X$. In this step, we choose the central polynomial to contain the denominator of $W_{1}$ and instead of the closed loop characteristic polynomial which is unknown, we put all its other roots on the origin, at $z=0$. Using the proposed method, in some steps we improve the central polynomial. We start with $\gamma_{S_{y p}}=60$ when only the constraint on $S_{y p}$ is employed. In the next step, based on the resulting controller $K=X Y^{-1}$ we choose a new central polynomial equal to the numerator of $W_{1}^{-1}(M Y+N X)$. Moreover, since the constraint on $S_{u p}$ is less violated, arbitrarily we use a fixed value $\gamma_{S_{u p}}=2$ for the bound on $S_{u p}$. Running the simulation program, we decrease $\gamma_{S_{y p}}$ in each step $\gamma_{S_{y p}}=5,3,2,1.8,1.4$ and we obtain two central polynomials for the constraints on $\gamma_{S_{y p}}$ and $\gamma_{S_{u p}}$. Note that in these steps we do not try to find the optimal $\gamma$ and just we decrease it in each step to find a better central polynomial without too much computational efforts. Then, using the iterative bisection algorithm the optimal $\gamma_{S_{y p}}=1.05$ is obtained, with criteria violation (see [33]) $J^{m}=\Delta S_{y p}+\Delta S_{u p}=4.01+0=4.01$. Figures 1 and 2 show the resulting closed-loop sensitivity functions, and the desired constraints. Moreover, The resulting controller is :

$$
K_{3}=\frac{0.014532(z+1)\left(z^{2}-1.162 z+1.397\right)}{(z-0.9466)\left(z^{2}-0.889 z+0.6669\right)}
$$




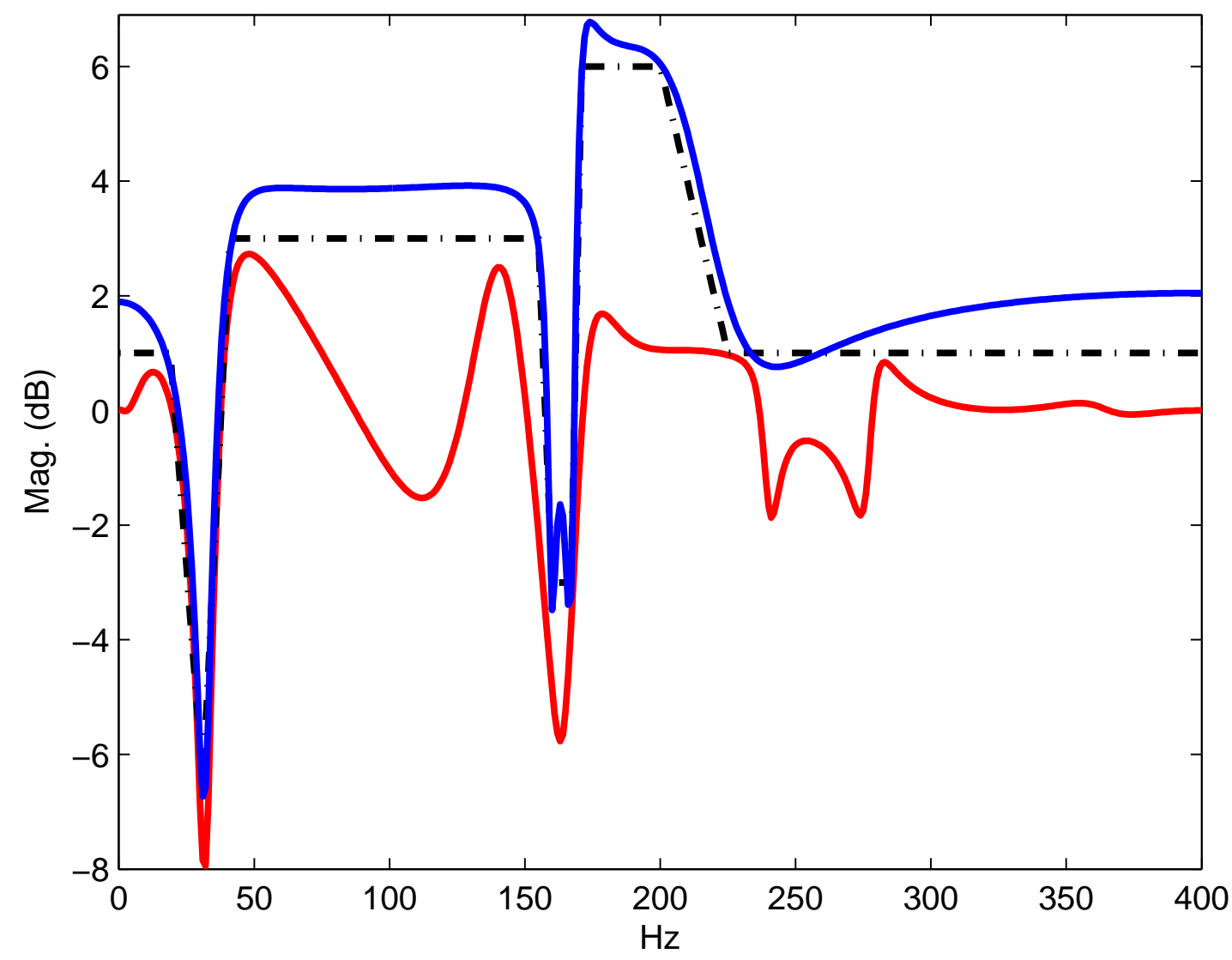

Fig. 1. The resulting $S_{y p}$ (Red), its desired constraint (dash-dot) and the inverse of the designed weighting function $W_{1}$ (Blue).

that can be considered as the second best controller in terms of criteria violation among those introduced in [33], however, with a less complexity index $C_{n}=7$, compared to $C_{n}=9$ for the best controller.

\section{B. Polytopic systems}

A very important feature of the proposed parameterization presented by (9) (or by (12)-(14)) is that it can be easily applied to the systems with polytopic uncertainty because in contrary to Youla parameterization, the system parameters are not involved in the controller parameterization. The following theorem extends this method for the polytopic systems.

Theorem 2: Consider the transfer functions of numerator and denominator of (6) for each 


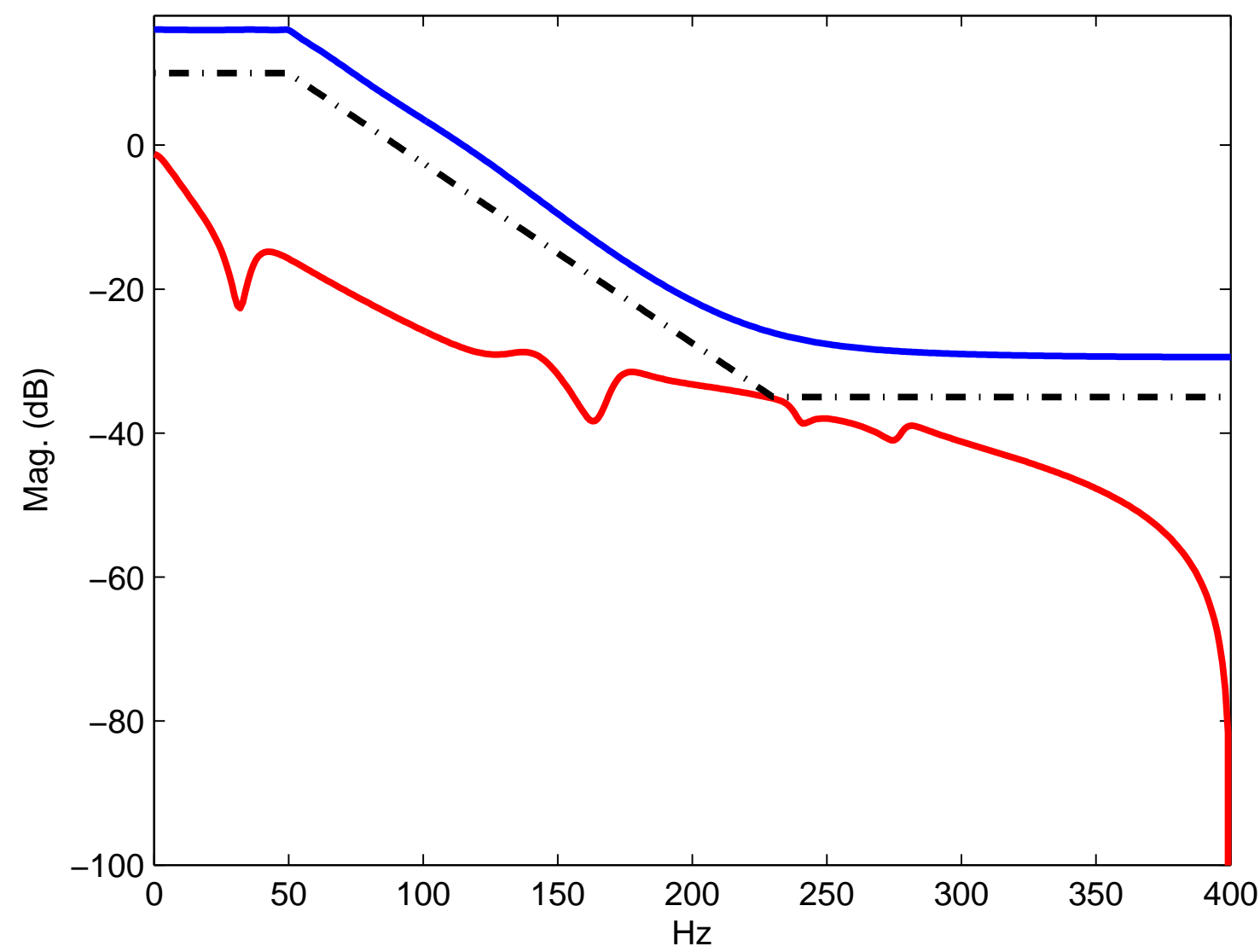

Fig. 2. The resulting $S_{u p}$ (Red), its desired constraint (dash-dot) and the inverse of the designed weighting function $W_{2}$ (Blue)

vertices of the system polytope defined in (2):

$$
\begin{aligned}
& \left(M_{i} Y+N_{i} X\right)-\gamma^{-1} W_{1} M_{i} Y \\
& \left(M_{i} Y+N_{i} X\right)+\gamma^{-1} W_{1} M_{i} Y
\end{aligned}
$$

Then, any controller belonging to the convex set :

$$
\mathscr{K}_{p_{\infty}}:\left\{K=X Y^{-1} \mid(18) \text { and (19) be CL-SPR, i=1,., q }\right\}
$$

where $X, Y \in \mathscr{R} \mathscr{H}_{\infty}$, stabilizes the polytopic system and results in a closed-loop polytopic system with $\left\|W_{1} S\right\|_{\infty}<\gamma$ for all of its members.

Proof: It should be shown that if $\left(M_{i} Y+N_{i} X\right)-\gamma^{-1} W_{1} M_{i} Y$ and $\left(M_{i} Y+N_{i} X\right)-\gamma^{-1} W_{1} M_{i} Y$ are CL-SPR for $i=1, \ldots, q$, then the controller $K=X Y^{-1}$ stabilizes the whole system polytope 
and in addition, satisfies $\left\|W_{1} S\right\|_{\infty}<\gamma$ for all members of the polytopic system $\mathscr{G}$ defined in (2). For each vertices of $\mathscr{G}$, the sum of two SPR transfer functions (18) and (19) results in the SPRness of $M_{i} Y+N_{i} X, i=1, \ldots, q$. In Th. 2 of [26], it is proved that such a controller stabilizes all members of $\mathscr{G}$. Next, we should show robust performance with this controller, i.e. it satisfies the $\mathscr{H}_{\infty}$ norm constraint for all members of $\mathscr{G}$. This is much easier to be shown via the LMI representation of (20):

$$
\begin{aligned}
\mathscr{K}_{p_{\infty}}:\{K= & X Y^{-1} \mid P_{i}=P_{i}^{T}>0, \\
& {\left[\begin{array}{cc}
A^{T} P_{i} A-P_{i} & A^{T} P_{i} B-C_{i_{1}}^{T} \\
B^{T} P_{i} A-C_{i_{1}} & B^{T} P_{i} B-D_{i_{1}}-D_{i_{1}}^{T}
\end{array}\right]<0 } \\
& {\left.\left[\begin{array}{cc}
A^{T} P_{i} A-P_{i} & A^{T} P_{i} B-C_{i_{2}}^{T} \\
B^{T} P_{i} A-C_{i_{2}} & B^{T} P_{i} B-D_{i_{2}}-D_{i_{2}}^{T}
\end{array}\right]<0, i=1, \ldots, q\right\} }
\end{aligned}
$$

where $\left(A, B, C_{i_{1}}, D_{i_{1}}\right)$ and $\left(A, B, C_{i_{2}}, D_{i_{2}}\right)$ are the controllable canonical state space realizations of (18) and (19), respectively. $A$ is assumed to be identical for all transfer functions (18) and (19) because of their identical denominators and $B$ is fixed because of the realization. Since all these constraints, i.e. (21) and (22), are linear w.r.t. the parameters of the system vertices, it is evident that any member of $\mathscr{G}$, i.e. $G=N M^{-1}$ such that $M=\sum_{i=1}^{q} \lambda_{i} M_{i}, N=\sum_{i=1}^{q} \lambda_{i} N_{i}, \lambda_{i} \geq 0$ and $\sum_{i=1}^{q} \lambda_{i}=1$, satisfies the LMIs (21) and (22) with $P=\sum_{i=1}^{q} \lambda_{i} P_{i}$ and therefore, satisfies $\left\|W_{1} S\right\|_{\infty}<\gamma$, too.

In other words, the proposed method ensures robust performance in addition to robust stability for the polytopic system.

\section{Remark :}

- Although the above theorem gives a sufficient condition and not a necessary and sufficient one, it is evident that by increasing the controller order, not only some controllers of new orders are included in $\mathscr{K}_{p_{\infty}}$, but also some controller of lower orders fall inside $\mathscr{K}_{p_{\infty}}$ by non-coprime $X$ and $Y$.

- Since we do not have a frequency interpretation for the CL-SPR constraint, the frequency gridding method of [26] is not applicable in this paper and only the LMI formulation can be used.

Example 2: For comparison purposes, the simulation example of [26] is studied. 


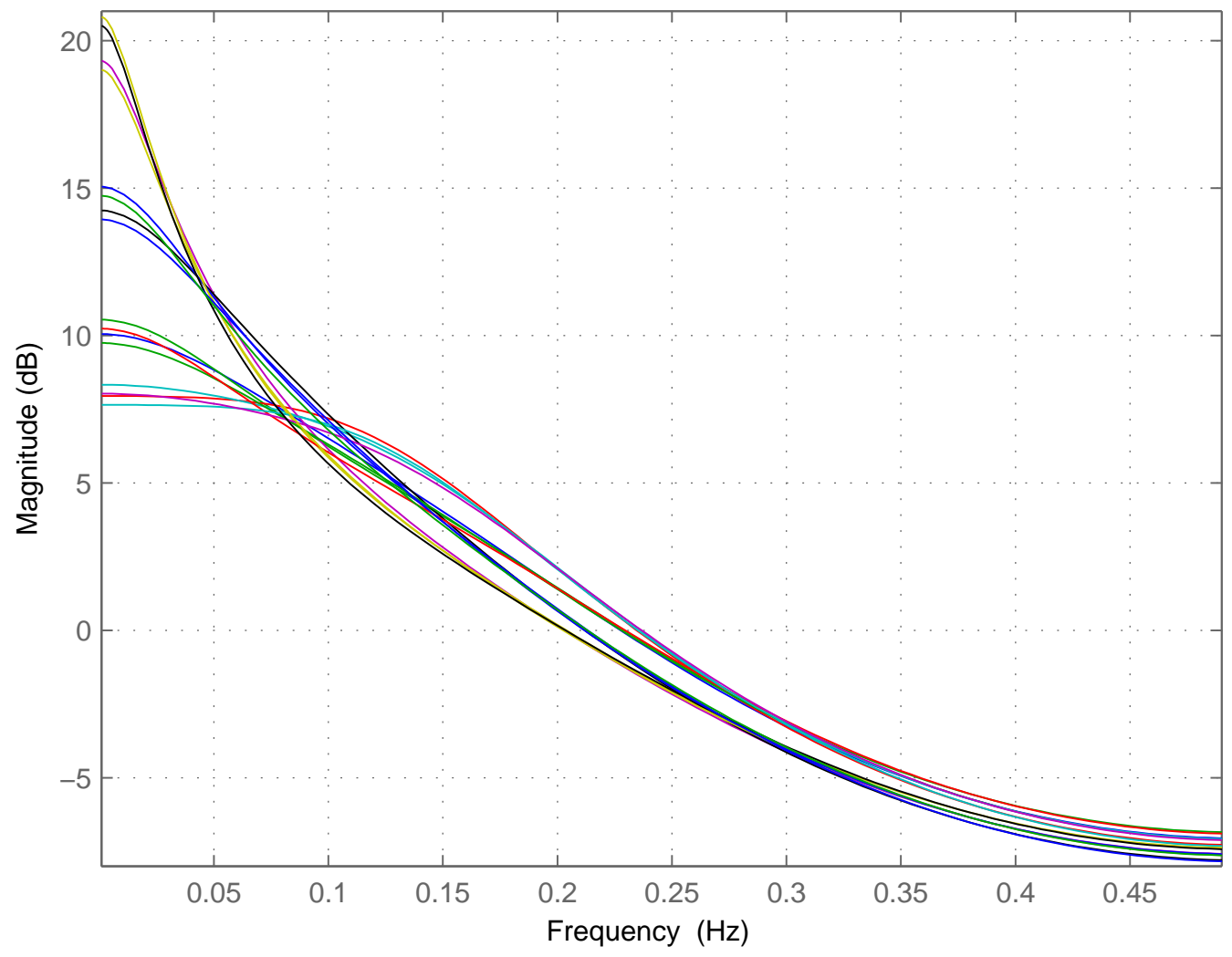

Fig. 3. Magnitude Bode diagrams of all vertices of the polytopic system.

Consider the problem of robust controller design for the following third-order system :

$$
G(z)=\frac{z+a}{z^{3}+b z^{2}+c z+d} \quad T_{s}=1 \mathrm{~s}
$$

with $a=0.2, b=-1.2, c=0.5$ and $d=-0.1$, where all the parameters are uncertain up to $\pm 7 \%$ of their nominal values, resulting in a four-dimensional hypercube with $2^{4}=16$ vertices. The magnitude Bode diagrams of all the 16 vertices of this polytope are depicted in Fig. 3. Large uncertainty in low frequencies shows that this is a tough system for robust control methods. Assume that the goal is to design a stabilizing controller that contains an integrator, and $\left\|W_{1} S\right\|_{\infty}$ should be minimized over all members of the polytopic system. The weighting function $W_{1}(z)$ 
is chosen to be the same as in [26] :

$$
W_{1}(z)=\frac{0.4902\left(z^{2}-1.0431 z+0.3263\right)}{z^{2}-1.282 z+0.282}
$$

which is a low-pass weighting filter based on the inverse of the desired sensitivity function. The same coprime factorization of [26] is used for the nominal plant model:

$$
\begin{aligned}
N & =\frac{z+0.2}{(z-0.1)\left(z^{2}-1.0431 z+0.3263\right)} \\
M & =\frac{z^{3}-1.2 z^{2}+0.5 z-0.1}{(z-0.1)\left(z^{2}-1.0432 z+0.3263\right)}
\end{aligned}
$$

Note that the denominator of all coprime factors are identical for all models in the polytopic system and $\zeta=0.1$ as in [26].

Before dealing with the polytopic system, we want to show the main advantage of the proposed method. That is, for a system without uncertainty, the proposed method can achieve the optimal $\mathscr{H}_{\infty}$ norm by increasing the controller order, irrelevant to the choice of the basis functions. Using the command hinfsyn for the first vertex of the polytopic system :

$$
G_{1}=\frac{z-.186}{z^{3}-1.116 z^{2}+0.465 z-0.093}
$$

we can have a fifth-order controller such that $\left\|W_{1} S\right\|_{\infty}=0.552$. Using basis functions with different $\zeta$, Fig. 4 shows that by increasing the controller order, the proposed method converges rapidly to the optimum norm bound of $\left\|W_{1} S\right\|_{\infty}$, independent of the basis function. Next, we design a controller for the polytopic system. The problem in [26] does not become feasible for controller orders less than 5 , because its approximation for $\mathscr{H}_{\infty}$ norm constraint has too much conservatism. However, using the proposed method of this paper, it becomes feasible with a second-order controller.

The controller $K_{2}=X Y^{-1}$ is parameterized as follows :

$$
\begin{aligned}
X & =\frac{x_{1} z^{2}+x_{2} z+x_{3}}{(z-0.1)^{2}} \\
Y & =\frac{(z-1)\left(y_{1} z+y_{2}\right)}{(z-0.1)^{2}}
\end{aligned}
$$

To solve the problem in MATLAB, YALMIP [34] is used as the interface and SDPT3 [35] as the solver. Using the iterative bisection algorithm, the optimal value of $\gamma_{o p t}=0.729$ is obtained with the following controller :

$$
K_{2}=\frac{0.802(z-0.6347)(z-0.1887)}{(z-1)(z+1.156)}
$$




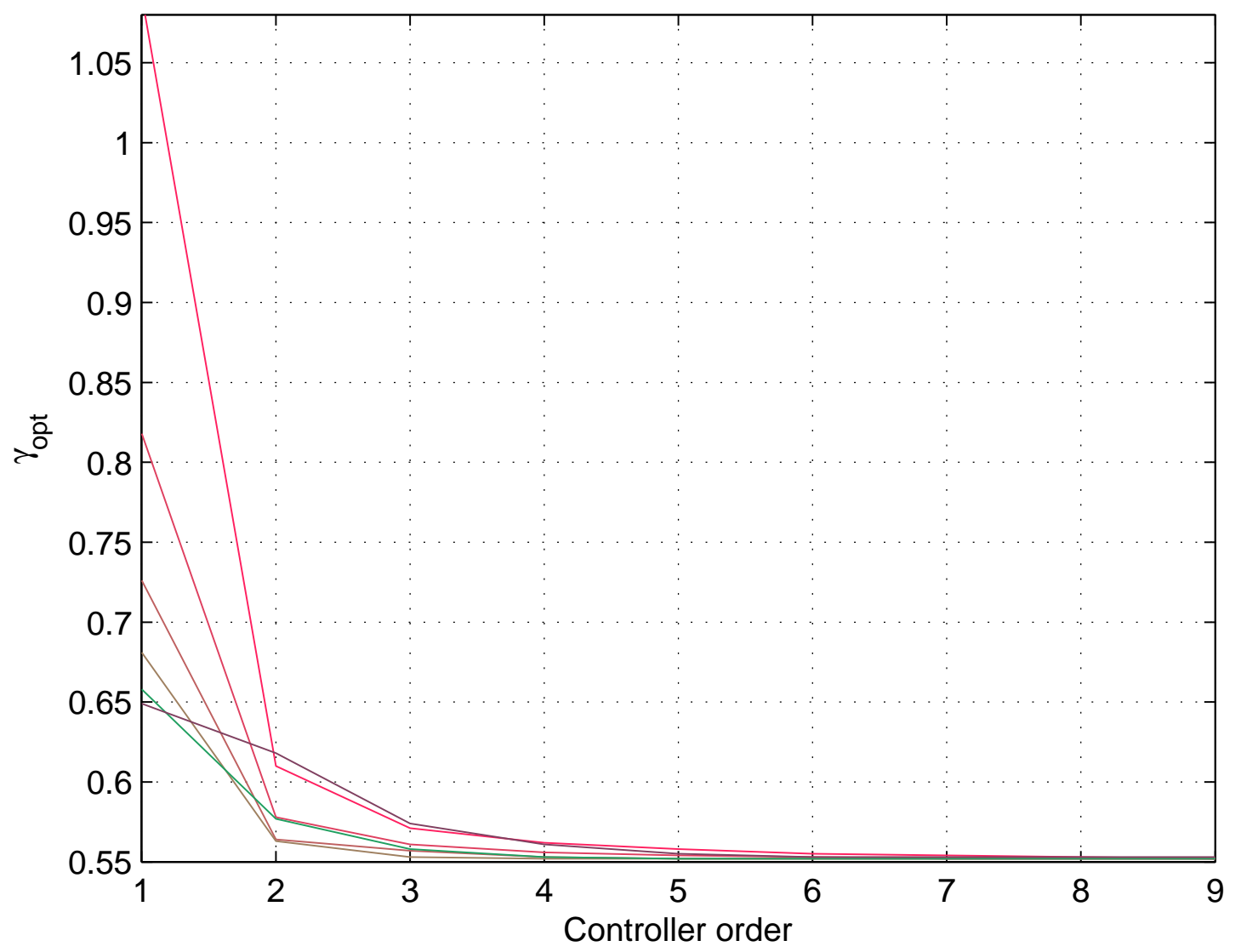

Fig. 4. $\gamma_{o p t}$ for the system 26 versus the order of the controller for different basis functions. Looking to the starting point from the highest curve, the basis function are chosen to have $\zeta=0,0.1,0.2,0.3,0.4,0.5$ respectively.

The magnitude Bode diagrams of $W_{1} S_{i}$ for all the 16 vertices of the polytopic system are shown in Fig. 5, where the $\gamma_{o p t}=0.729=-2.7454 d B$ is also depicted. Furthermore, their sensitivity functions are shown in Fig. 6 in addition to the Bode magnitude diagram of $\gamma_{o p t} / W_{1}$. The maximum value of the sensitivity functions is around $5.3 \mathrm{~dB}$, which is quite desirable [29].

\section{Remark :}

- The above method designs an $\mathscr{H}_{\infty}$ controller for a polytopic system, which means that both unstrauctured and structured uncertainties are considered in this design method.

- Since the state space realization has been employed the extension of the above results to MIMO systems is quite straightforward. The only difference is that for MIMO systems such as for polytopic systems, even when the system has no uncertainty, the proposed method 


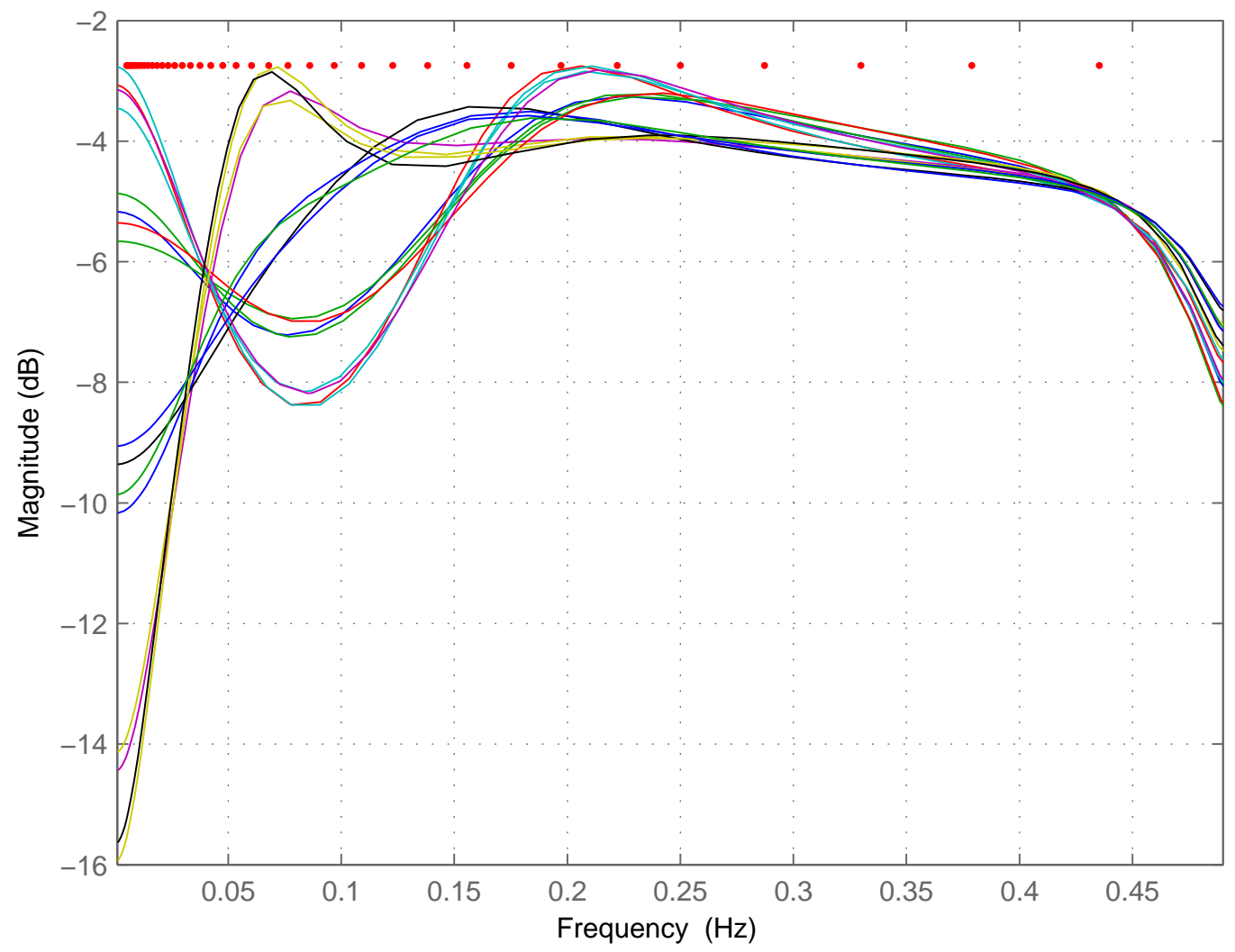

Fig. 5. Bode magnitude diagram of $W_{1} S_{i}$ of all vertices of the polytopic system with the second-order controller (29) (Solid) and $\gamma_{o p t}$ (Dotted).

gives an inner convex approximation of the set of all $\mathscr{H}_{\infty}$ controllers.

The disadvantage of the proposed method is that it may result in a high-order controller (the same disadvantage of [26]), which is not practical for many real systems.

\section{REDUCED-ORDER CONTROLLER DESIGN}

As mentioned in the above section, to cope with the conservatism due to the fixation of basis functions, the order of the controller is relaxed. Thus, to achieve the optimal $\gamma$, the resulting controller is generally of high order. In the following, a set of convex constraints and a new convex cost function will be added to the problem in order to force the solver to find a controller 


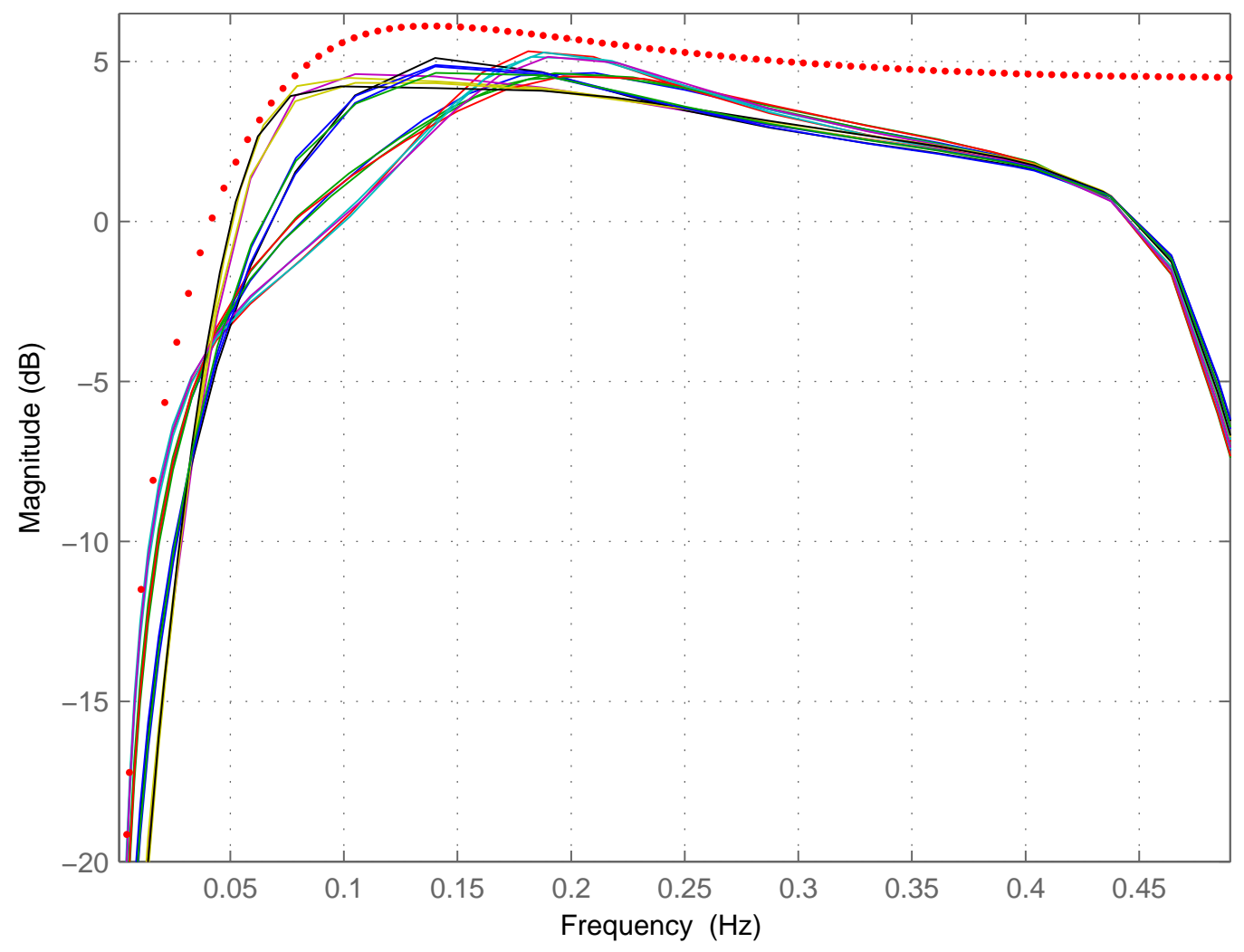

Fig. 6. Output sensitivity function of all vertices of the polytopic system with the second-order controller (29) (Solid), Bode magnitude diagram of $\gamma_{o p t} / W_{1}$ (Dotted).

that has a desired number of pole-zero cancellation. This way, the resulting controller will be of the desired order.

\section{A. Sylvester resultant}

It is shown that the number of common roots between two $m$-th order polynomials $x=$ $x_{m} z^{m}+x_{m-1} z^{m-1}+\cdots+x_{0}$ and $y=z^{m}+y_{m-1} z^{m-1}+\cdots+y_{0}$ is equal to the rank deficiency of their first Sylvester resultant matrix [36]. Let the $k$-th Sylvester resultant matrix $S_{k}$ be defined 
as follows :

$$
S_{k}=\left[\begin{array}{cccccc}
1 & & & x_{m} & & \\
y_{m-1} & \ddots & & x_{m-1} & \ddots & \\
\vdots & \ddots & 1 & \vdots & \ddots & x_{m} \\
y_{1} & \ddots & y_{m-1} & x_{1} & \ddots & x_{m-1} \\
y_{0} & \ddots & \vdots & x_{0} & \ddots & \vdots \\
& \ddots & y_{1} & & \ddots & x_{1} \\
& & y_{0} & & x_{m-(k-1)}
\end{array}\right]
$$

where $1 \leq k \leq m$. Taking into account the structure of $S_{k}$, it is obvious that the rank deficiency of $S_{1}$ is strongly related to that of $S_{k}$, such that $\operatorname{Rank}\left(S_{1}\right) \leq 2 m-k$ if and only if $S_{k}$ is not full rank (see [37] and [38] (Th. 2.3)).

Suppose that $x$ and $y$ are the polynomials of numerator and denominator of a controller such that $K=X Y^{-1}=x / y$. Therefore, to force a controller of order $m$ to have $k$ zero-pole cancellation, the rank of matrix $S_{1}$ should be $2 m-k$, which in turn means that $S_{k}$ should be rank-deficient.

Rank constraints are non-convex and an optimization containing such constraints has been shown to be NP-hard [39], [40]. However, instead of rank minimization, we need only the $S_{k}$ to be rank-deficient. The rank deficiency of $S_{k}$ can be easily represented via a bilinear matrix equality (BME). Defining $s_{k}^{1}$ as the first column of $S_{k}$ and $\hat{S}_{k}$ such that $S_{k}=\left[s_{k}^{1} \hat{S}_{k}\right]$, then $S_{k}$ is rank-deficient if and only if there exists a vector $u$, such that $\hat{S}_{k} u=s_{k}^{1}$. Therefore, the following feasibility problem parameterizes all fixed-order stabilizing $\mathscr{H}_{\infty}$ controllers of order $(m-k)$ for the system $G$ defined in (1) :

$$
\begin{cases}\exists u \in \mathscr{R}^{2 m-2 k+1 \times 1} \text { such that: } & \hat{S}_{k} u=s_{k}^{1} \\ \exists P=P^{T}>0 \text { such that: } & \text { (7) and (8) be CL-SPR }\end{cases}
$$

where, $x, y, u$ and $P$ are the variables. Obviously, the equality constraint is not convex, due to the multiplication of the variables in the left hand side. Thus, we need to find a convex approximation of this BME problem to be able to solve it efficiently. 


\section{B. Convex approximation of rank deficiency}

There are very few results in convexification of the rank constraints in the literature. A wellknown cost function to minimize the rank of a symmetric positive semi-definite matrix is its trace, which is linear w.r.t. the matrix elements and is equal to the $\ell_{1}$-norm of the vector of its eigenvalues. It is quite obvious that to force a vector to become sparse, minimizing its $\ell_{1}$ norm is the best, compared to the other $\ell_{p}$-norms. Fortunately, we need just to make a matrix rank-deficient and there is no need to really minimize it. However, the related matrix, $S_{k}$ is not a symmetric positive semi-definite matrix. Thus, $S_{k}^{T} S_{k}$ is an appropriate candidate, which is a symmetric positive semi-definite matrix and its rank is equal to the rank of $S_{k}$ [41]. The trace of $S_{k}^{T} S_{k}$, which is the $\ell_{1}$-norm of its eigenvalues, is proportional to the $\ell_{2}$-norm of the controller parameters, i.e.

$$
\operatorname{trace}\left(S_{k}^{T} S_{k}\right)=(m-k+1)\left[1+\sum_{i=1}^{m} y_{i}^{2}+\sum_{j=0}^{m} x_{j}^{2}\right]
$$

This quadratic cost function is a convex function and can be minimized to force pole-zero cancellation in the controller. To append this cost function to (9), for a rather high-order controller, first we find the optimal $\gamma_{o p t}$ using iterative bisection algorithm and then, we fix a $\gamma_{0}$ such that $\gamma_{o p t}<\gamma_{0}$, in order to have a larger feasible set. Then, the following optimization problem is employed :

$$
\operatorname{Minimize}\left(\sum_{i=1}^{m} y_{i}^{2}+\sum_{j=0}^{m} x_{j}^{2}\right)
$$

Subject to: CL-SPR constraint on

$$
(M Y+N X)-\gamma_{0}{ }^{-1} W_{1} M Y \text { and }(M Y+N X)+\gamma_{0}{ }^{-1} W_{1} M Y
$$

This problem will be referred to as Direct problem, because it minimizes the trace of $S_{k}^{T} S_{k}$ directly.

Remark : In Direct method (32), $k$ does not have any role, because the trace of $S_{k}^{T} S_{k}$ and $S_{1}^{T} S_{1}$ are the same up to a fixed multiplier. Therefore, there is no control on the number of pole-zero cancellation in the controller.

This quadratic cost function of (32) can be converted to a linear cost function using the following lemma [15] : 
Lemma 4: Rank $S_{k} \leq \ell$ if and only if there exists symmetric positive semi-definite matrices $U$ and $V$ such that :

$$
\operatorname{Rank} U+\operatorname{Rank} V \leq 2 \ell, \quad\left[\begin{array}{cc}
U & S_{k} \\
S_{k}^{T} & V
\end{array}\right] \geq 0
$$

According to this lemma, decreasing the rank of $U$ and $V$, leads to decrement in rank of $S_{k}$. The rank of $U$ and $V$ can possibly be decreased by minimizing trace $(U)+\operatorname{trace}(V)$. This cost function will be referred to as Embedded, since the above lemma is called the Embedded lemma in [15]. The advantage of minimizing this linear cost function rather than $\operatorname{trace}\left(S_{k}^{T} S_{k}\right)$ is that the optimization problem becomes an SDP and can be solved more reliably via existing solvers such as SeDuMi [42]. Furthermore, in contrast to Direct problem, the desired number of pole-zero cancellation in the controller, i.e. $k$, enters in the problem by the size of $U$ and $V$. The following optimization problem uses the above lemma :

$$
\begin{aligned}
& \text { Minimize }\left(\sum_{i=1}^{2 m-k+1} U(i, i)+\sum_{j=1}^{2(m-k+1)} V(j, j)\right), \text { Subject to: } \\
& U=U^{T}, V=V^{T} \geq 0,\left[\begin{array}{cc}
U & S_{k} \\
S_{k}^{T} & V
\end{array}\right] \geq 0 \text { and } \\
& \text { CL-SPR constraint on }(M Y+N X)-\gamma_{0}{ }^{-1} W_{1} M Y \text { and }(M Y+N X)+\gamma_{0}{ }^{-1} W_{1} M Y
\end{aligned}
$$

Remark : Minimizing the trace of a symmetric semi-definite matrix is just an approximation for minimizing its rank, i.e. a symmetric positive semi-definite matrix with a small trace is not necessarily rank-deficient or even close to it. The condition number, which is equal to the ratio of maximum to minimum singular value, is an appropriate index of the rank deficiency of a matrix. On the other hand, the trace of a symmetric positive semi-definite matrix is not only equal to the $\ell_{1}$-norm of the vector of its eigenvalues, but also is equal to the $\ell_{1}$-norm of its diagonals. Besides, if a diagonal becomes zero, an eigenvalue becomes zero too. Thus, one way to produce zero eigenvalues, i.e. to have a rank-deficient matrix, is to try to have a sparse vector of diagonals. One way to accomplish such a task, i.e. to force the solver to produce zero diagonals, is to modify Embedded cost function from $\operatorname{trace}(U)+\operatorname{trace}(V)=\sum_{i=1}^{2 m-k+1} U(i, i)+\sum_{j=1}^{2(m-k+1)} V(j, j)$ to Weighted-Embedded $\sum_{i=1}^{2 m-k+1} w^{i} U(i, i)+\sum_{j=1}^{2(m-k+1)} w^{j} V(j, j)$, where $0<w \leq 1$ inserts less weight on some of the diagonals, in order to move the others towards zero. Therefore, we can 
TABLE I

COMPARISON OF THE DIFFERENT RANK MINIMIZATION METHODS

\begin{tabular}{|c|c|c|c|c|}
\hline Method & BME & Direct & Emb & WEmb \\
\hline Condition number & $1.3 e 6$ & 5.6 & 38 & 79 \\
\hline Linsearch steps & 471 & 46 & 61 & 99 \\
\hline
\end{tabular}

minimize Weighted-Embedded cost function in (34), in order to increase the chance of having rank deficient $S_{k}$.

In the following example, the effectiveness of the rank-deficiency constraint on Sylvester resultant matrix of two polynomials is checked via different convex approximations of the rank minimization problem.

Example 3: Consider two ninth-order polynomials $a$ and $b$ with some unknown parameters. The goal is to see if it is possible to force them to have a desired number of common roots by means of the rank-deficiency constraint on their Sylvester resultant matrix. Let $\left[\begin{array}{lllllllll}1 & a_{1} & 1.6 & a_{2} & 0.5 & a_{3} & a_{4} & a_{5} & a_{6}\end{array}\right]$ and $\left[\begin{array}{lllllllll}1 & 2 & b_{1} & 1 & b_{2} & 0.5 & b_{3} & 0.3 & b_{4}\end{array}\right]$ be the parameter vectors of the polynomials $a$ and $b$, respectively, where $a_{i}$ and $b_{i}$ are the optimization variables. The objective is to make the fourth Sylvester resultant matrix of $a$ and $b$ rank-deficient, which means that they should have four common roots. Table (I) shows the condition number of the Sylvester matrix for different methods and also the computational effort of each method. The distances between the common roots of polynomials $a$ and $b$ with BME method is of order $1 e-12$ whereas for Weighted-Embedded method the distances are 8.6e-4 and 5.0e-3. For Embedded method there is only one pair of common roots with distance of $3.8 e-3$ and in Direct method there is only one common root related to the case where $a_{6}=b_{4}=0$.

The root map of the resulting polynomials is shown in Fig. 7 for BME method and in Fig. 8 for Embedded method. Furthermore, the root map of Weighted-Embedded method is shown in Fig. 9. It is obvious that Weighted-Embedded method works better than Embedded method. The chosen weight in this example is $w=0.5$. Moreover, the computational time in this simple problem is very short, thus the number of Linsearch steps is chosen in order to compare the computational effort. Table (I) shows that the convex approximations need much less computational effort than the non-convex BME method.

Example 4: Here we want to design a third-order controller for the same system $G_{1}$ in (26). 


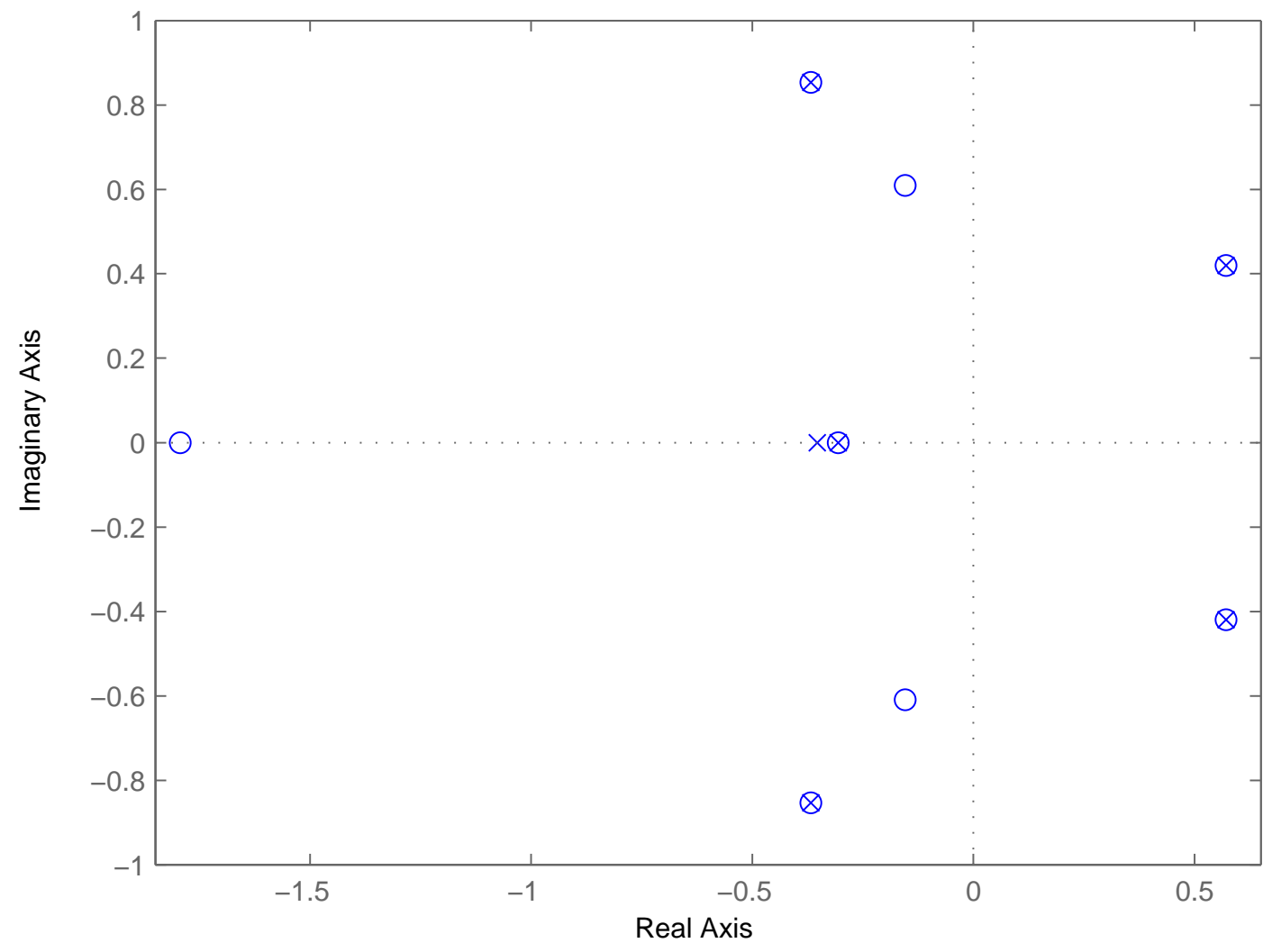

Fig. 7. Roots of polynomials $a$ and $b$ with BME method.

The difference is that we want a third-order controller that achieves the optimal $\gamma_{\text {opt }}=0.552$. Employing the same basis functions and the same weight of Ex. 2, $\gamma_{o p t}=0.562$ is obtained with the following third-order controller :

$$
K_{3}=\frac{0.55822(z-0.4918)(z+0.3254)(z-0.09174)}{(z-1)(z+1.037)(z+0.4923)}
$$

Then, we increase the controller order. For a 15 -th order controller, $\gamma_{o p t .}=0.552$ is obtained. To have a bigger feasible set we choose $\gamma_{0}=0.553$ and use the problem of (34) with WeightedEmbedded cost function and with $k=12$ and $w=0.95$. Pole-zero map of the 15-th order controller is shown in Fig. 10. Twelve pole-zero cancellation are clearly observed. The reducedorder controller :

$$
K_{3_{r}}=\frac{0.55754(z-0.5991)\left(z^{2}-0.4585 z+0.1427\right)}{(z-1)(z+1.095)(z-0.3338)}
$$




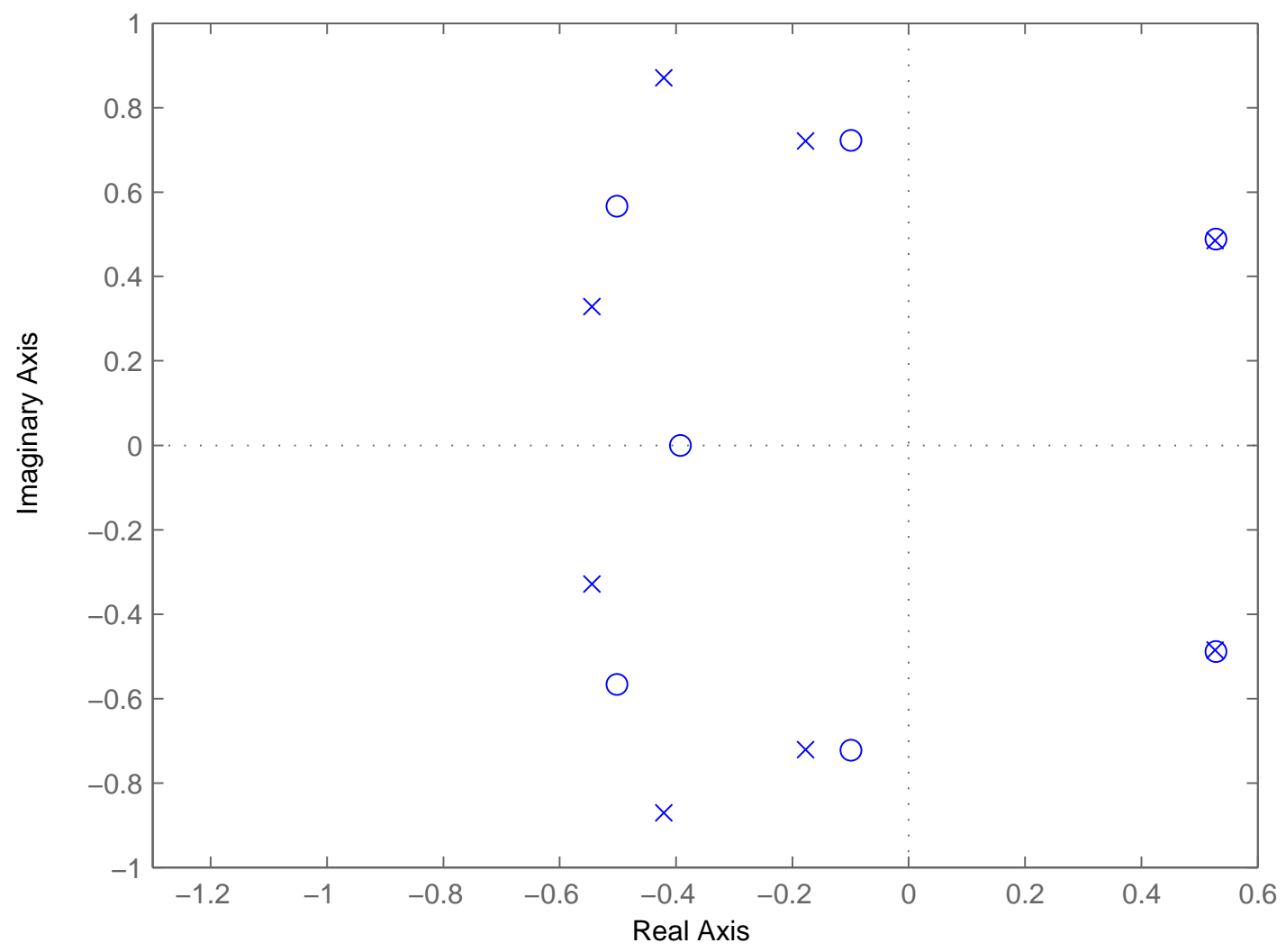

Fig. 8. Roots of polynomials $a$ and $b$ with Embedded method.

achieves $\left\|W_{1} S\right\|_{\infty}=0.552$ and hence, it is clear that it does not belong to (9) with its third order $X$ and $Y$, whereas it belongs to (9) with its 15-th order $X$ and $Y$. This example shows that appending the rank deficiency constraint on the Sylvester matrix of the controller to the proposed parameterization (9) can remove the disadvantage of resulting in high-order controllers.

\section{Conclusions}

A new convex parameterization of all $\mathscr{H}_{\infty}$ stabilizing controllers for SISO-LTI systems is given based on a new concept of Common Lyapunov Strictly Positive Realness. This convex parameterization provides a complete set of all $\mathscr{H}_{\infty}$ controllers of a single system. Moreover, it can be used for low-order controller design and can deal with any controller structure such as e.g. PID, whereas with Youla parameterization it is not possible to enforce any controller structure 


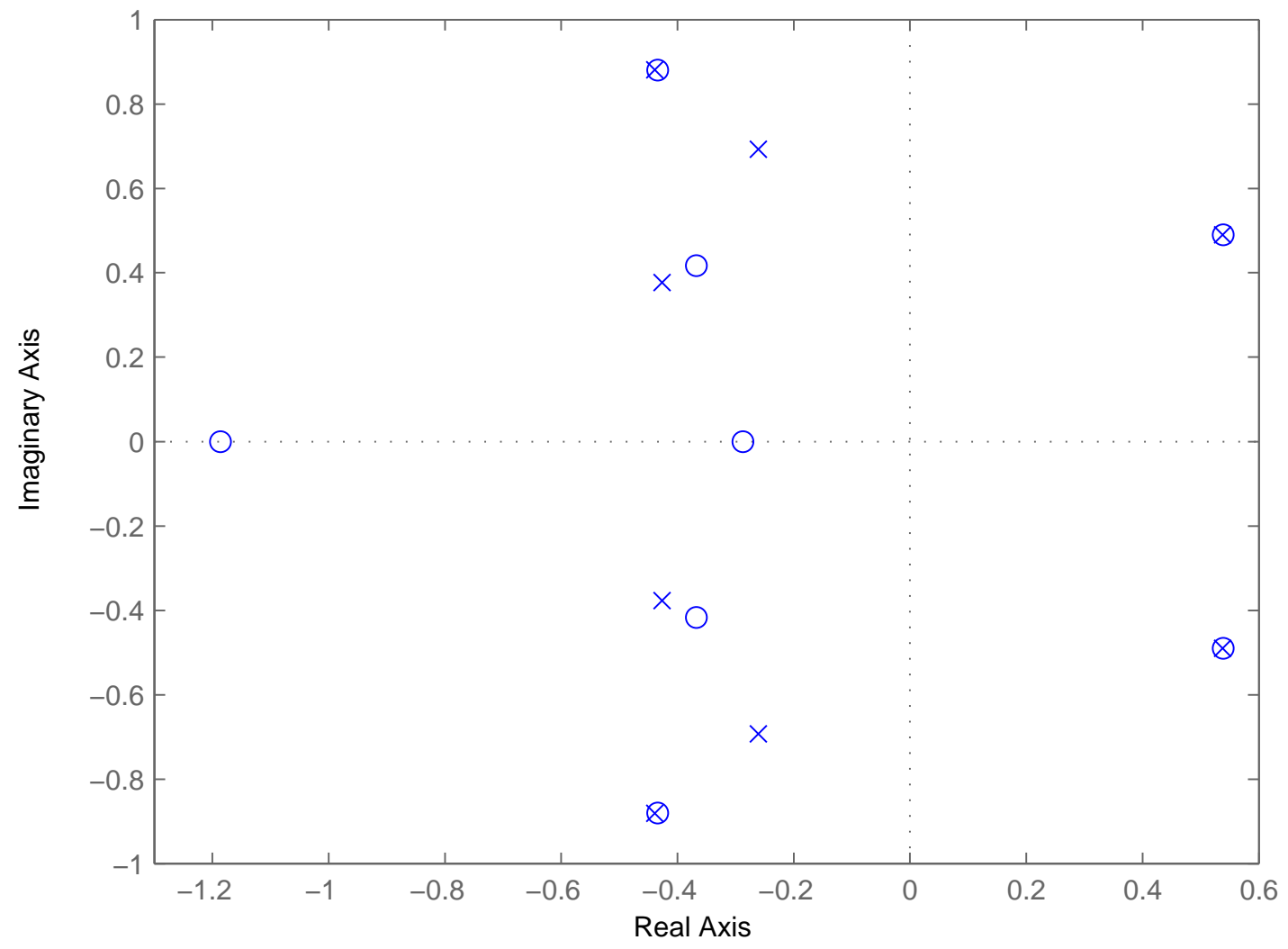

Fig. 9. Roots of polynomials $a$ and $b$ with Weighted-Embedded method.

and orders lower than that of the system. Furthermore, the proposed method is straightforwardly employed for the systems with polytopic uncertainty. The proposed method normally results in a high-order controller. Using a convexified rank deficiency constraint on the Sylvester matrix of the controller parameters, the solver tries to find a controller with the desired number of pole-zero cancellation. This way, a convex inner approximation of the non-convex set of all fixed-order $\mathscr{H}_{\infty}$ controllers is developed, which is larger than the resulting inner approximations of [10] and [8].

\section{REFERENCES}

[1] C. J. Doyle, B. A. Francis, and A. R. Tannenbaum, Feedback Control Theory. New York: Mc Millan, 1992. 


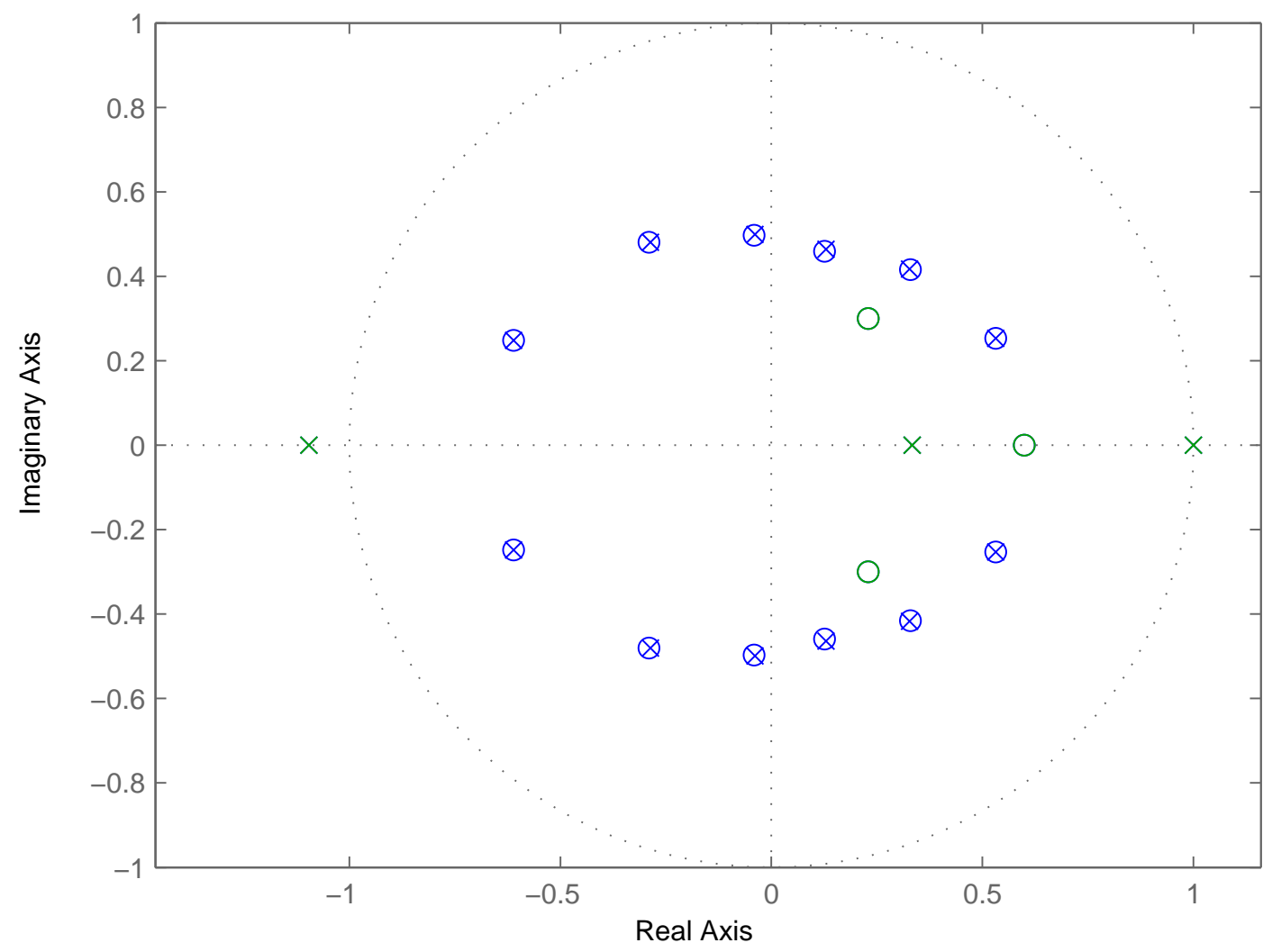

Fig. 10. Pole-zero map of the 15 -th order controller. Obviously there are 12 pole-zero cancellation.

[2] W. S. Levine and M. Athans, "On the determination of the optimal constant output feedback gains for linear multivariable systems," IEEE Transactions on Automatic Control, vol. 15, no. 1, pp. 44-48, February 1970.

[3] J. Ackermann, Robust Control: Systems with Uncertain Physical Parameters. New York, NY: Springer Verlag, 1993.

[4] M. G. Safonov, K. C. Goh, and J. H. Ly, "Control system synthesis via bilinear matrix inequalities," in IEEE American Control Conference, Baltimore. Maryland, 1994.

[5] O. Toker and H. Ozbay, "On the NP-hardness of solving bilinear matrix inequalities and simultaneous stabilization with static output feedback," in IEEE American Control Conference, Seattle, Washington, 1995.

[6] T. Iwasaki and R. E. Skelton, "All controllers for the general $h_{\infty}$ control problem - LMI existence conditions and state space formulas," Automatica, vol. 30, no. 8, pp. 1307-1317, 1994.

[7] S. Wang and J. H. Chow, "Low-order controller design for SISO systems using coprime factors and LMI," IEEE Transactions on Automatic Control, vol. 45, no. 6, pp. 1166-1169, June 2000.

[8] D. Henrion, M. Sebek, and V. Kucera, "Positive polynomials and robust stabilization with fixed-order controllers," IEEE Transactions on Automatic Control, vol. 48, no. 7, pp. 1178-1186, 2003. 
[9] J.-K. Shiau and C.-E. Tseng, "A discrete $H_{\infty}$ low-order controller design using coprime factors," Tamkang Journal of Science and Engineering, vol. 7, no. 4, pp. 251-258, 2004.

[10] H. Khatibi, A. Karimi, and R. Longchamp, "Fixed-order controller design for systems with polytopic uncertainty using lmis," IEEE Transactions on Automatic Control, vol. 53, no. 1, pp. 428-434, 2008.

[11] C. W. J. Hol, C. W. Scherer, E. G. van der Meche, and O. H. Bosgra, "A nonlinear SDP approach to fixed-order controller synthesis and comparison with two other methods applied to an active suspension system," European Journal of Control, vol. 9, no. 1, pp. 13-28, June 2003.

[12] D. Henrion, S. Tarbouriech, and M. Sebek, "Rank-one LMI approach to simultaneous stabilization of linear systems," System and Control Letters, vol. 38, pp. 79-89, 1999.

[13] P. Gahinet and P. Apkarian, "A linear matrix inequality approach to $H_{\infty}$ control," International Journal of Robust and Nonlinear Control, vol. 4, pp. 421-448, 1994.

[14] M. Mesbahi and G. P. Papavassilopoulos, "On the rank minimization problem over a positive semi-definite linear matrix inequality," IEEE Transactions on Automatic Control, vol. 42, no. 2, pp. 239-243, February 1997.

[15] M. Fazel, H. Hindi, and S. P. Boyd, "Log-det heuristic for matrix rank minimization with applications to Hankel and Euclidean distance matrices," in American Control Conference, Denver, Colorado, 2003.

[16] S. P. Bhattacharyya, H. Chapellat, and L. H. Keel, Robust Control: The Parametric Approach. NJ, USA: Prentice Hall Information and System Sciences Series, 1995.

[17] B. R. Barmish, New Tools for Robustness of Linear Systems. New York, NY, 1994: Macmillan, 1994.

[18] E. Feron, P. Apkarian, and P. Gahinet, "Analysis and synthesis of robust control systems via parameter-dependent Lyapunov functions," IEEE Transactions on Automatic Control, vol. 41, no. 7, pp. 1041-1047, 1996.

[19] D. Peaucelle, D. Arzelier, O. Bachelier, and J. Bernussou, "A new robust D-stability condition for real convex polytopic uncertainty," Systems and Control Letters, vol. 40, pp. 21-30, 2000.

[20] D. Henrion, D. Arzelier, D. Peaucelle, and J. B. Lasserre, "On parameter-dependent Lyapunov functions for robust stability of linear systems," in 43rd IEEE Conference on Decision and Control, Atlantis, Paradise Island, Bahamas, December 2004, pp. 887-892.

[21] G. Chesi, A. Garulli, A. Tesi, and A. Vicino, "An LMI-based technique for robust stability analysis of linear systems with polynomial parametric uncertainties," in Lecture Notes in Control and Information Sciences. Heidelberg: Springer-Verlag, 2005, vol. 312 .

[22] K. Zhou, Essentials of Robust Control. New Jersey: Prentice Hall, 1998.

[23] Y. Ebihara and T. Hagiwara, "Robust controller synthesis with parameter-dependent Lyapunov variables: A dilated LMI approach," in 41st IEEE Conference on Decision and Control, Las Vegas, NV, USA, December 2002, pp. 4179-4184.

[24] P. Apkarian, H. D. Tuan, and J. Bernussou, "Continuous-time analysis, eigenstructure assignment, and $H_{2}$ synthesis with enhanced linear matrix inequalities (LMI) characterizations," IEEE Transactions on Automatic Control, vol. 46, no. 12, pp. 1941-1946, December 2001.

[25] A. Rantzer and A. Megretski, "Convex parameterization of robustly stabilizing controllers," IEEE Transactions on Automatic Control, vol. 39, no. 9, pp. 1802-1808, September 1994.

[26] A. Karimi, H. Khatibi, and R. Longchamp, "Robust control of polytopic systems by convex optimization," Automatica, vol. 43, no. 6, pp. 1395-1402, 2007.

[27] F. Yang, M. Gani, and D. Henrion, "Fixed-order robust $H_{\infty}$ controller design with regional pole assignment," IEEE Transactions on Automatic Control, vol. 52, no. 10, pp. 1959-1963, 2007. 
[28] R. Wallin, A. Hansson, and L. Vandenberghe, Efficiently solving semidefinite programs originating from the KalmanYakubovich-Popov lemma using general purpose SDP solvers. http://www.control.isy.liu.se/publications: Technical report from the Control and Communication group in Linkoping, Report number: LiTH-ISY-R-2503, 2003.

[29] I. D. Landau, R. Lozano, and M. M’Saad, Adaptive Control. London: Springer-Verlag, 1997.

[30] C. Scherer, P. Gahinet, and M. Chilali, "Multiobjective output-feedback control via LMI optimization," IEEE Transactions on Automatic Control, vol. 42, no. 7, pp. 896-911, July 1997.

[31] B. D. O. Anderson, "The small-gain theorem, the passivity theorem and their equivalence," J. Franklin Inst., vol. 293, no. 2, pp. 105-115, Februry 1972.

[32] Z. S. Duan, L. Huang, and L. Wang, "Multiplier design for extended strict positive realness and its applications," International Journal of Control, vol. 77, no. 17, pp. 1493-1502, November 2004.

[33] I. D. Landau, A. Karimi, L. Mišković, and H. Prochazka, "Control of an active suspension system as a benchmark for design and optimisation of restricted-complexity controlers," European Journal of Control, vol. 9, no. 1, pp. 3-12, January 2003.

[34] J. Löfberg, "YALMIP: A toolbox for modeling and optimization in MATLAB," in CACSD Conference, 2004. [Online]. Available: http://control.ee.ethz.ch/ joloef/yalmip.php

[35] K. C. Toh, M. J. Todd, and R. H. Tutuncu, "SDPT3: a MATLAB software package for semidefinite programming," Optimization Methods and Software, vol. 11, pp. 545-581, 1999.

[36] C. T. Chen, Linear System Theory and Design. New York, USA: Oxford, 1999.

[37] D. Rupperecht, "An algorithm for computing certified approximate GCD of $n$ univariate polynomials," Journal of Pure and Applied Algebra, vol. 139, pp. 255-284, 1999.

[38] E. Kaltofen, Z. Yang, and L. Zhi, "Structured low rank approximation of a Sylvester matrix," in International Workshop on Symbolic-Numeric Computation SNC 2005 Proceedings, Xian, China, July 19-21 2005, pp. 188-201.

[39] L. Vandenberghe and S. Boyd, "Semidefinite programming," SIAM Reviews, vol. 38, no. 1, pp. 49-95, March 1996.

[40] B. Recht, M. Fazel, and P. A. Parrilo, "Guaranteed minimum-rank solutions of linear matrix equations via nuclear norm minimization," 2007. [Online]. Available: http://arxiv.org/abs/0706.4138

[41] W. M. Haddad and D. S. Bernstein, "Robust stabilization with positive real uncertainty: Beyond the small gain theorem," in 29th IEEE Conference on Decision and Control, Honolulu, Hawaii, 1990, pp. 2054-2059.

[42] J. F. Sturm, "Using SeDuMi 1.02, a Matlab toolbox for optimization over symmetric cones," Optimization Methods and Software, vol. 11, pp. 625-653, 1999.

[43] S. P. Boyd, L. El Ghaoui, E. Feron, and V. Balakrishnan, Linear Matrix Inequalities in System and Control Theory. SIAM, 1994.

\section{APPENDIX}

In case of continuous-time systems, it is possible to remove the multiplication between the controller parameters and $\gamma$ in the dual equations of (13) and (14) (and (21) and (22)). This way, we can minimize $\gamma$ in our convex optimization problem without an iterative bisection algorithm. Let the biproper transfer functions $(M Y+N X)+\gamma^{-1} W_{1} M Y$ and $(M Y+N X)-\gamma^{-1} W_{1} M Y$ have controllable canonical state space realizations $\left(A, B, C_{1}, D_{1}\right)$ and $\left(A, B, C_{2}, D_{2}\right)$ respectively. Since 
$W_{1}$ is strictly proper [1], $\gamma$ appears just in $C_{i}=\hat{C}_{i}+\gamma^{-1} \tilde{C}_{i} i=1,2$ and not in $D_{1}$ and $D_{2}$. Obviously, $\hat{C_{1}}=\hat{C_{2}}=\hat{C}$ and $\tilde{C}_{1}=-\tilde{C_{2}}=\tilde{C}$. Moreover, for a strictly proper system $D_{1}=D_{2}=D$. Taking into account the KYP Lemma for the continuous-time systems [43], and imposing the CL-SPR constraint (9) : $\exists P=P^{T}>0$ such that

$$
\begin{aligned}
& {\left[\begin{array}{cc}
A^{T} P+P A & P B-\left(\hat{C}+\gamma^{-1} \tilde{C}\right)^{T} \\
B^{T} P-\left(\hat{C}+\gamma^{-1} \tilde{C}\right) & -D-D^{T}
\end{array}\right]<0} \\
& {\left[\begin{array}{cc}
A^{T} P+P A & P B-\left(\hat{C}-\gamma^{-1} \tilde{C}\right)^{T} \\
B^{T} P-\left(\hat{C}-\gamma^{-1} \tilde{C}\right) & -D-D^{T}
\end{array}\right]<0}
\end{aligned}
$$

Since $(N X+M Y)+\gamma^{-1} W_{1} M Y$ is biproper, $D+D^{T}$ is invertible. Using the inverse of Schur complement, (37) is equivalent to :

$$
A^{T} P+P A+\left(P B-\hat{C}^{T}-\gamma^{-1} \tilde{C}^{T}\right)\left(D+D^{T}\right)^{-1}\left(B^{T} P-\hat{C}-\gamma^{-1} \tilde{C}\right)<0
$$

To simplify the notations, let $Q=A^{T} P+P A$ and $V=P B-\hat{C}^{T}$ and $R=\left(D+D^{T}\right)$. Therefore, (39) is equivalent to :

$$
Q+V R^{-1} V^{T}+\gamma^{-2} \tilde{C}^{T} R^{-1} \tilde{C}-\gamma^{-1} \tilde{C}^{T} R^{-1} V^{T}-\gamma^{-1} V R^{-1} \tilde{C}<0
$$

which in turn is equal to :

$$
\tilde{Q}+\tilde{C}^{T}\left(-\gamma^{-1} R^{-1}\right) V^{T}+V\left(-\gamma^{-1} R^{-1}\right) \tilde{C}<0
$$

where $\tilde{Q}=Q+V R^{-1} V^{T}+\gamma^{-2} \tilde{C}^{T} R^{-1} \tilde{C}$. Since $R$ is fixed, $\gamma^{-1} R^{-1}$ contains no controller variables. Therefore, using Finsler's lemma [43], this constraint becomes equivalent to :

$$
\exists \sigma \in \mathbb{R} \text { s.t. }
$$

$$
\begin{aligned}
& \tilde{Q}+\sigma \tilde{C}^{T} \tilde{C}<0 \\
& \tilde{Q}+\sigma V V^{T}<0
\end{aligned}
$$

This way, the multiplication of $\gamma^{-1}$ with other variables can be removed from the constraints. Using Schur complement three times, the new constraints (40) and (41) can be represented by 
the following LMIs :

$$
\begin{aligned}
& {\left[\begin{array}{cccc}
A^{T} P+P A & P B-\hat{C}^{T} & \tilde{C}^{T} & P B-\hat{C}^{T} \\
B^{T} P-\hat{C} & -D-D^{T} & 0 & 0 \\
\tilde{C} & 0 & -\eta\left(D+D^{T}\right) & 0 \\
B^{T} P-\hat{C} & 0 & 0 & -\mu I
\end{array}\right]<0} \\
& {\left[\begin{array}{cccc}
A^{T} P+P A & P B-\hat{C}^{T} & \tilde{C}^{T} & \tilde{C}^{T} \\
B^{T} P-\hat{C} & -D-D^{T} & 0 & 0 \\
\tilde{C} & 0 & -\eta\left(D+D^{T}\right) & 0 \\
\tilde{C}^{T} & 0 & 0 & -\mu I
\end{array}\right]<0}
\end{aligned}
$$

where $\eta=\gamma^{2}$ and $\mu=\sigma^{-1}$. These inequalities represent an LMI version of (37). By changing the sign of $\tilde{C}$, similar LMIs can be derived for (38). Since $\tilde{C}$ appears symmetrically in (42) and (43), its sign does not change the determinant of any of the leading principal minors of these matrices and hence, it is sufficient to satisfy these two LMIs and there is no need for the other ones. Hence, the set of all controllers given by (6) can be represented by (42) and (43) and $\eta=\gamma^{2}$ can be minimized. 